\title{
ON THE APPLICABILITY OF THE AUXILIARY SYSTEM APPROACH IN COMPLEX NETWORKS WITH THE RING TOPOLOGY
}

\author{
Volodymyr Lynnyk, Branislav Rehák, Sergej Čelikovský \\ The Czech Academy of Sciences \\ Institute of Information Theory and Automation, Czech Republic \\ voldemar@utia.cas.cz; rehakb@utia.cas.cz; celikovs@utia.cas.cz
}

Article history:

Received 15.10.2019, Accepted 26.11.2019

\begin{abstract}
The generalized synchronization of the complex network consisting of nodes being the chaotic systems via the auxiliary system approach is studied here. More specifically, the applicability of the auxiliary system approach to detect the generalized synchronization in the complex network consisting of unidirectional coupled chaotic systems in the cyclic chain is investigated. Such a complex network has the so-called ring topology. It is shown that the auxiliary system approach is useful for this purpose. The presented analysis is supported by the numerical simulations as well.
\end{abstract}

\section{Key words}

Complex networks, nonlinear systems, synchronization, chaos.

\section{Introduction}

Complex networks have been studied within many different branches of science [Chen et al., 2014]. Complex network $(\mathrm{CN})$ structure is usually represented the graph consisting of nodes (vertices) coupled by links (edges). Many real-world and man-made systems can be described by complex networks, e.g. Internet, World Wide Web, metabolic networks, biological neural networks, collaboration networks, food webs, electric power grids [Khramenkov et al., 2019] etc. Traditionally, complex networks were studied via classical graph theory and random graph theory introduced by [Erdös and Rényi, 1960], nevertheless, these basics were significantly extended during the last two decades. Furthermore, there has been an increasing interest in the synchronization of coupled systems as well. The synchronization is a significant phenomenon in nature an as such has been studied by many scientists. Indeed, it is one of the simplest but still very important types of the collective dynam- ics of the interconnected systems. Studies of the identical (complete, full) synchronization of complex systems were started by the papers [Fujisaka and Yamada, 1983; Pikovsky, 1984] and the analysis of the synchronization phenomena between the chaotic coupled systems was rapidly started thanks to the paper [Pecora and Carroll, 1990]. In case of the identical synchronization (IS), the states of the interconnected systems to be synchronized should mutually converge each to other. The so-called master-slave synchronization [Pecora and Carroll, 1990] is used in analog/digital communication for the chaotic masking, see [Čelikovský and Lynnyk, 2016] and the references therein.

Besides the IS approach, many other kinds of synchronizations of the interconnected systems have been introduced recently, such as: phase synchronization (PS) [Rosenblum et al., 1996], generalized synchronization (GS) [Rulkov et al., 1995; Kocarev and Parlitz, 1996], projective synchronization [Mainieri and Rehacek, 1999], lag synchronization (LS) [Rosenblum et al., 1997], $\varepsilon$-synchronization [Plotnikov and Fradkov, 2019], etc. The quality and the speed of the synchronization of the coupled systems depend not only on the structure of the network but also on the presence of different types of disturbances in the communication channels, e.g. the limited-band data erase [Andrievsky, 2016], time delays [Klinshov and Nekorkin, 2017; Rehák and Lynnyk, 2019; Rehák and Lynnyk, 2019], noise [Moskalenko et al., 2017], quantization [Andrievsky et al., 2017; Rehák and Lynnyk, 2019] etc.

The general knowledge about different types of synchronization between coupled chaotic systems and complex networks can be found in a comprehensive review [Boccaletti et al., 2002] and the books [Chen and Dong, 1998; Boccaletti et al., 2018].

The present paper is devoted to the GS approach. GS is characterized by the existence of the functional relation- 


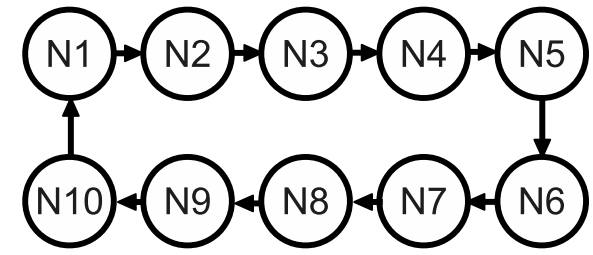

Figure 1. Directed complex network $C_{10}$ with a ring topology.

ship between the interconnected units. The GS of the unidirectional coupled systems has been first introduced by [Afraimovich et al., 1986]. Later, in [Rulkov et al., 1995], the notion of the GS was coined and the GS between two unidirectional connected units was detected using the mutual false nearest neighbors method. In addition, in [Abarbanel et al., 1996], the so-called auxiliary system approach to detect the GS was introduced. In this context, the main contribution of the present paper is the study of the generalized synchronization phenomenon of a class of complex network where all nodes are identical chaotic systems with different control parameters and initial conditions. The motivation of the current research is to show that the auxiliary system approach can be used to detect the generalized synchronization phenomena in the complex network with unidirectional coupling between nodes and ring topology. More specifically, the complex network studied here consist of the nodes being the so-called generalized Lorenz system (GLS). It was already shown in [Čelikovský and Chen, 2005] that two GLS's, though both being three-dimensional systems, with the master-slave structure can be identically synchronized using the single scalar connection only. The identical synchronization in complex network with bidirectionally connected nodes being the GLS with the same control parameter and different initial conditions was obtained in [Čelikovský et al., 2007]. Moreover, the robustness in the dynamical complex networks with different topologies was rigorously studied in [Čelikovský et al., 2013].

In such a way, the present paper will continue the research started in [Čelikovský et al., 2013], namely, it will address the detection of the generalized synchronization in complex network having the ring topology. It will show that in the case, when every node of the unidirectional complex network has different behaviors, the auxiliary system approach allows detecting the GS phenomenon. In other words, the novelty of this paper is the testing the applicability of the auxiliary system approach to unidirectional coupled systems with chain connections. Note, that in auxiliary system approach, the number of auxiliary systems is equal to the total number of the nodes in the analyzed complex network.

The rest of the paper is organized as follows. Some facts about complex networks and synchronization phenomena are introduced in the next section. Preliminaries about generalized Lorenz system and the synchronization in the complex networks consisting of the nodes being the GLS are introduced in Section 3. Section 4 re- peats some knowledge about auxiliary system approach used later on to detect the GS regime in the directed cyclic complex networks. Verification of the detection method via numerical simulations is provided in Section 5. Conclusions and outlooks are given in the final section.

\section{Synchronization in Complex Networks}

\subsection{The generalized Synchronization}

The generalized synchronization (GS) is a kind of synchronization, for which there exists a functional relationship between coupled systems. To be more specific, consider two different unidirectional interconnected systems or two similar systems with a different control parameter (parameters). These systems are given by the vector fields $f$ and $g$, where $g$ includes the coupling

$$
\begin{aligned}
& \dot{\eta}=f(\eta) \\
& \dot{\hat{\eta}}=g(\hat{\eta}, \eta) .
\end{aligned}
$$

Here, $\eta \in \mathbb{R}^{n}$ and $\hat{\eta} \in \mathbb{R}^{m}$ denote the states of the drive (master) system and the response (slave) systems [Parlitz, 2012]. Systems (1a) and (1b) are synchronized if there exists a continuous function $\Phi: \mathbb{R}^{n} \mapsto \mathbb{R}^{m}$ mapping the states of the drive into the state space of the response system. The properties of this transformation $\Phi$ do not depend upon the initial conditions in the basin of attraction of the chaotic attractor [Rulkov et al., 1995]. The orbits of the overall system lie in a subspace of the full state space $\mathbb{R}^{n} \oplus \mathbb{R}^{m}$. Systems (1a) and (1b) are said to be mutually $\mathbf{G S}$ if

$$
\lim _{t \rightarrow \infty}(\hat{\eta}(t)-\Phi(\eta(t))=0 .
$$

Furthermore, the necessary and sufficient condition of the GS is the existence of the asymptotically stable limit set of the response system [Kocarev and Parlitz, 1996].

\subsection{The Identical Synchronization of Networks}

The identical synchronization (IS) of the master-slave configuration (1) is the particular case of the GS when $\Phi$ equals to the identity transformation.

Next, let us introduce the IS for a more general configurations forming the complex network (CN). Namely, consider the complex network having $N$ identical nodes being nonlinear sytems. Each node is described by

$$
\dot{\eta^{i}}=f\left(\eta^{i}\right)+\sum_{j=1}^{N} a_{j i} \phi\left(\eta^{i}, h\left(\eta^{j}\right), L\right),
$$

where $\eta^{i}=\left(\eta_{1}, \eta_{2}, \ldots, \eta_{n},\right)^{\top} \in \mathbb{R}^{n}$ is the state vector of node $i, i=1, \ldots, N, L=\left(l_{1}, l_{2}, \ldots, l_{n}\right)^{\top}$ is the vector of coupling gains, $h(\cdot)$ is a scalar synchronizing output of each system, $\phi$ is nonlinear coupling with $\phi(\eta, h(\eta), L) \equiv 0 \forall \eta, L$ and $A=\left(a_{i j}\right)_{i, j=1, \ldots, N}$ 


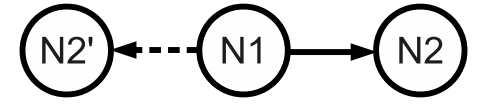

Figure 2. Block scheme of the auxiliary system approach to detect GS of the unidirectional coupled nonlinear oscillators proposed by [Abarbanel et al., 1996]. Here, N1 is a drive (master) system, N2 is a response (slave) system and $\mathrm{N} 2$ ' is auxiliary system which is a copy of the response system N2. N2 and N2' are driven by the same signal.

is the so-called adjacency matrix that has no loops, i.e. $i \neq j$. Here, $a_{i j}$ is not always equal to $a_{j i}$, because the graph is directed, i.e. the adjacency matrix $A$ may be nonsymmetric. Without any loss of generality one can set $a_{i i}=0 \forall i \in\{1,2, \ldots, N\}$, due to the above assumption that $\phi(\eta, h(\eta), L) \equiv 0 \forall \eta, L$. Network (3) is said to be identically (asymptotically) synchronized if, $\forall i, j \in\{1,2, \ldots, N\}$ :

$$
\lim _{t \rightarrow \infty}\left(\eta^{i}(t)-\eta^{j}(t)\right)=0 .
$$

Definition 2.1. The $C N(3)$ is said to be synchronizable if there exists an integer $\mu \in\{1,2, \ldots, N\}$, such that for every $\sigma \in\{1,2, \ldots, N\}$ there exists a sequence of integers $\left\{\kappa_{1}, \ldots, \kappa_{l}\right\}$ satisfying

$$
\kappa_{1}=\mu, \kappa_{l}=\sigma, a_{\kappa_{1}, \kappa_{2}}=\cdots=a_{\kappa_{l-1}, \kappa_{l}}=1 .
$$

If the above integer $\mu$ is unique, then the node with number $\mu$ is called the master of CN (3). The synchronizable network is called minimal, if removing any connection makes it not synchronizable. Obviously, a minimal synchronizable network always has a master [Čelikovský et al., 2013].

In other words, the synchronizable network is related to a directed graph that contains rooted directed spanning tree, where the root is a master. Moreover, if a complex network is a rooted directed spanning tree, then the $\mathrm{CN}$ is a minimal one.

\subsection{Directed Cyclic Complex Network}

Complex network $(\mathrm{CN})$ with the topology described by the cycle (circle) graph is called cyclic complex network $(\mathrm{CCN})$. In computer networks theory this kind of topology is usually called the ring topology. This kind of $\mathrm{CN}$ consists of some number of nodes connected in a closed chain. The CCN with $N$ nodes will be denoted $C_{n}$. The number of links in CCN equals the number of nodes. Every node in the $\mathrm{CCN}$ has obviously the degree (number of connected links to the node) equal to 2.

A directed cyclic $\mathbf{C N}$ (DCCN) is the directed version of CCN. Here, all links are being oriented in the same direction. DCCN has uniform in-degree (number of incoming links into each node) 1 and uniform out-degree (number of outgoing links emanating from each node) 1. Adjacency matrix $A$ of DCCN is a cyclic matrix (i.e., each its row and column has precisely one nonzero entry). Fig. 1 shows a DCCN $C_{10}$ consisting of ten nodes.
In [Čelikovský et al., 2013] it was noted that the DCCN is always synchronizable, but never minimal.

One can expect that if any (D)CCN subnetwork of a given general $\mathrm{CN}$ is synchronized then so can be whole $\mathrm{CN}$. This clearly motivates the study of (D)CCN.

\section{Generalized Lorenz Systems and Their Identical Synchronization in Complex Networks}

The complex network with nodes being the generalized Lorenz system (GLS) is studied here. GLS generalizes the classical Lorenz system containing GLS as a particular case with some specific parameters choice.

Definition 3.1. [Čelikovský and Chen, 2002] The following general nonlinear system of ordinary differential equations in $\mathbb{R}^{3}$ is called GLS:

$$
\dot{x}=\left[\begin{array}{cc}
A & 0 \\
0 & \lambda_{3}
\end{array}\right] x+\left[\begin{array}{c}
0 \\
-x_{1} x_{3} \\
x_{1} x_{2}
\end{array}\right], A=\left[\begin{array}{ll}
a_{11} & a_{12} \\
a_{21} & a_{22}
\end{array}\right]
$$

where $x=\left[\begin{array}{lll}x_{1} & x_{2} & x_{3}\end{array}\right]^{\top}, \lambda_{3} \in \mathbb{R}$, and $A$ has the eigenvalues $\lambda_{1}, \lambda_{2} \in \mathbb{R}$, such that

$$
-\lambda_{2}>\lambda_{1}>-\lambda_{3}>0 .
$$

GLS is said to be nontrivial if it has a solution that goes neither to zero nor to infinity nor to a limit cycle.

The inequality (6) among the eigenvalues of the approximate linearization of GLS at the origin corresponds the well-known Shilnikov's conditions of the existence of chaos near homoclinic orbits of the saddle-point [Shilnikov, 1968; Shilnikov et al., 1993]. A canonical form of GLS is introduced by the following theorem.

Theorem 3.2. [Čelikovsky and Chen, 2002] For the nontrivial GLS (5) - (6), there exists a linear change of coordinates, $z=T x$ transforming (5) into the following generalized Lorenz canonical form (GLCF]:

$$
\dot{z}=\left[\begin{array}{ccc}
\lambda_{1} & 0 & 0 \\
0 & \lambda_{2} & 0 \\
0 & 0 & \lambda_{3}
\end{array}\right] z+c z\left[\begin{array}{ccc}
0 & 0 & -1 \\
0 & 0 & -1 \\
1 & \tau & 0
\end{array}\right] z
$$

where $z=\left[z_{1}, z_{2}, z_{3}\right]^{\top}, c=[1,-1,0], \tau \in(-1, \infty)$.

The parameter $\tau$ plays important role of the single scalar bifurcation parameter, while remaining parameters has only the qualitative influence being eigenvalues of the approximate linearization of GLS at the origin. These qualitative parameters are just required to satisfy robust condition (6), so that fine numerical tuning may be done using the single scalar parameter $\tau$ only. As a consequence, GLCF enables to generate rich collection of the parameterized chaotic behaviors [Čelikovský and Chen, 2002]. Furthermore, let us review yet another canonical form of GLS given by the following theorem. 


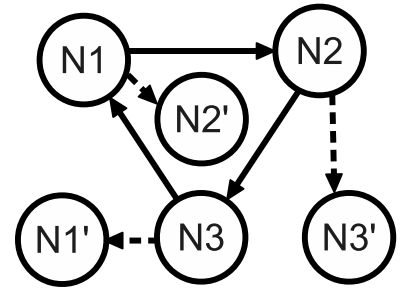

Figure 3. The topology of the three-nodes directed cyclic complex network with three additional auxiliary nodes $N 1^{\prime}, N 2^{\prime}$ and $N 3^{\prime}$ using for the detection of the GS regime.
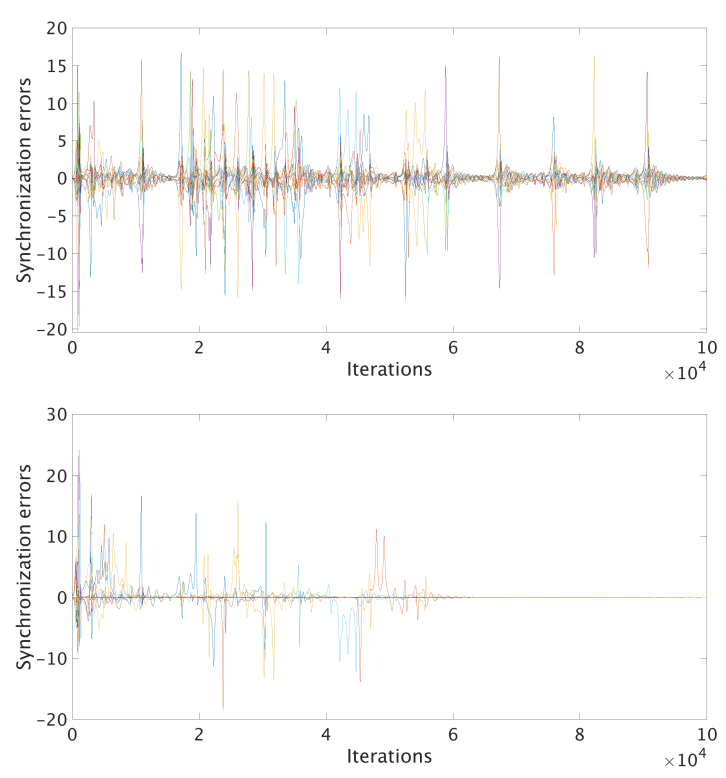

Figure 4. Synchronization error of 10-node complex network with ring topology and directional coupling (top subfigure). Synchronization error between permanent and auxiliary nodes in a directed cyclic complex network (bottom subfigure). The topology of this complex network it shown at Fig. 1.

Theorem 3.3. [Čelikovský and Chen, 2005] GLS is state equivalent to the following system, further referred to as the GLS observer canonical form (GLSOCF):

$$
\begin{gathered}
\frac{\mathrm{d} \eta}{\mathrm{d} t}=\left[\begin{array}{c}
\left(\lambda_{1}+\lambda_{2}\right) \eta_{1}+\eta_{2} \\
-\lambda_{1} \lambda_{2} \eta_{1}-\left(\lambda_{1}-\lambda_{2}\right) \eta_{1} \eta_{3}-\frac{(\tau+1)}{2} \eta_{1}^{3} \\
\lambda_{3} \eta_{3}+K_{\tau} \eta_{1}^{2}
\end{array}\right], \\
K_{\tau}=\frac{\lambda_{3}(\tau+1)-2 \tau \lambda_{1}-2 \lambda_{2}}{2\left(\lambda_{1}-\lambda_{2}\right)} .
\end{gathered}
$$

where $\eta=\left[\eta_{1}, \eta_{2}, \eta_{3}\right]^{\top}$. The corresponding smooth coordinate change and its inverse are

$$
\eta=\left[\begin{array}{c}
z_{1}-z_{2} \\
\lambda_{1} z_{2}-\lambda_{2} z_{1} \\
z_{3}-\frac{(\tau+1)\left(z_{1}-z_{2}\right)^{2}}{2\left(\lambda_{1}-\lambda_{2}\right)}
\end{array}\right], z=\left[\begin{array}{c}
\frac{\lambda_{1} \eta_{1}+\eta_{2}}{\lambda_{1}-\lambda_{2}} \\
\frac{\lambda_{2} \eta_{1}+\eta_{2}}{\lambda_{1}-\lambda_{2}} \\
\eta_{3}+\frac{(\tau+1) \eta_{1}^{2}}{2\left(\lambda_{1}-\lambda_{2}\right)}
\end{array}\right] .
$$

Note, that GLSOCF contains only four parameters, where $\lambda_{1,2,3}$ are the eigenvalues of the system linearization (7) and control parameter $\tau$ enables fine chaos tuning. Furthermore, , when viewing $\eta_{1}=x_{1}=$ $z_{1}-z_{2}$ as the output, GLSOCF resembles the wellknown linearizable by output injection form. As a consequence, GLSOCF provides the master-slave synchronization of two coupled GLS's using only scalar signal $\eta_{1}$ [Čelikovský and Chen, 2005]. Moreover, GLSOCF enables the synchronization of the pair of bidirectionally coupled GLS's, as shown by the following theorem.

Theorem 3.4. [Čelikovský et al., 2013]. Consider the CN formed by the pair of bidirectionally coupled systems in GLSOCF (8)-(9) having the states $\eta, \hat{\eta}$ and outputs $\eta_{1}, \hat{\eta}_{1}$ :

$$
\begin{aligned}
& \frac{\mathrm{d} \eta}{\mathrm{d} t}=\left[\begin{array}{c}
\left(\lambda_{1}+\lambda_{2}\right) \eta_{1}+\eta_{2} \\
-\lambda_{1} \lambda_{2} \eta_{1}-\left(\lambda_{1}-\lambda_{2}\right) \eta_{1} \eta_{3}-\frac{(\tau+1)}{2} \eta_{1}^{3} \\
\lambda_{3} \eta_{3}+K_{\tau} \eta_{1}^{2}
\end{array}\right] \\
& +a_{21}\left(\left[\begin{array}{c}
\left(\lambda_{1}+\lambda_{2}\right) \eta_{1} \\
-\lambda_{1} \lambda_{2} \eta_{1}-\left(\lambda_{1}-\lambda_{2}\right) \eta_{1} \eta_{3}-\frac{(\tau+1)}{2} \eta_{1}^{3} \\
K_{\tau} \eta_{1}^{2}
\end{array}\right]\right. \\
& -\left[\begin{array}{c}
\left(\lambda_{1}+\lambda_{2}\right) \hat{\eta}_{1} \\
-\lambda_{1} \lambda_{2} \hat{\eta}_{1}-\left(\lambda_{1}-\lambda_{2}\right) \hat{\eta}_{1} \eta_{3}-\frac{(\tau+1)}{2} \hat{\eta}_{1}^{3} \\
K_{\tau} \hat{\eta}_{1}^{2}
\end{array}\right] \\
& \left.+\left[\begin{array}{c}
l_{1}\left(\eta_{1}-\hat{\eta}_{1}\right) \\
l_{2}\left(\eta_{1}-\hat{\eta}_{1}\right) \\
0
\end{array}\right]\right) \text {, } \\
& \frac{\mathrm{d} \hat{\eta}}{\mathrm{d} t}=\left[\begin{array}{c}
\left(\lambda_{1}+\lambda_{2}\right) \hat{\eta}_{1}+\hat{\eta}_{2} \\
-\lambda_{1} \lambda_{2} \hat{\eta}_{1}-\left(\lambda_{1}-\lambda_{2}\right) \hat{\eta}_{1} \hat{\eta}_{3}-\frac{(\tau+1)}{2} \hat{\eta}_{1}^{3} \\
\lambda_{3} \hat{\eta}_{3}+K_{\tau} \hat{\eta}_{1}^{2}
\end{array}\right]
\end{aligned}
$$

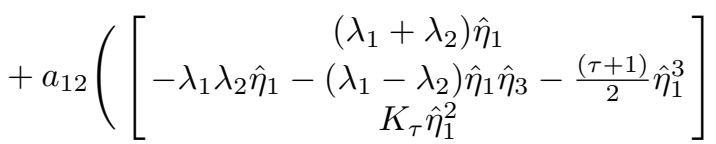

$$
\begin{aligned}
& -\left[\begin{array}{c}
\left(\lambda_{1}+\lambda_{2}\right) \eta_{1} \\
-\lambda_{1} \lambda_{2} \eta_{1}-\left(\lambda_{1}-\lambda_{2}\right) \eta_{1} \hat{\eta}_{3}-\frac{(\tau+1)}{2} \eta_{1}^{3} \\
K_{\tau} \eta_{1}^{2}
\end{array}\right] \\
& \left.+\left[\begin{array}{c}
l_{1}\left(\hat{\eta}_{1}-\eta_{1}\right) \\
l_{2}\left(\hat{\eta}_{1}-\eta_{1}\right) \\
0
\end{array}\right]\right) \text {, }
\end{aligned}
$$

where $l_{1,2}<0$ are synchronizing gains and let the solution of (10) with $a_{21}=0$ is bounded. Then it holds:

1. for $a_{12}=0, a_{21}=1$ or $a_{21}=0, a_{12}=1$, and for all gains $l_{1,2}<0$, one has $\lim _{t \rightarrow \infty}(\eta(t)-\hat{\eta}(t))=0$ globally and exponentially;

2. for $a_{12}=1, a_{21}=1$, and for every bounded region of initial conditions of system (10)-(11), there exist sufficiently large gains $l_{1,2}<0$ such that $\lim _{t \rightarrow \infty}(\eta(t)-\hat{\eta}(t))=0$. 
Note, that (10) and (11) are clearly in the form of CN (3) having the adjacency matrix

$$
A=\left[\begin{array}{cc}
0 & a_{12} \\
a_{21} & 0
\end{array}\right] .
$$

The synchronizing connections are the expressions in the large round brackets multiplied by $a_{12}$ and $a_{21}$.

Next, consider CN consisting of $N$ nodes of GLSOCF:

$$
\begin{aligned}
& {\left[\begin{array}{c}
\eta_{1}^{i} \\
\eta_{2}^{i} \\
\eta_{3}^{i}
\end{array}\right]=\left[\begin{array}{c}
\frac{\left(\lambda_{1}+\lambda_{2}\right) \eta_{1}^{i}+\eta_{2}^{i}}{\overline{-\lambda_{1} \lambda_{2} \eta_{1}^{i}-\left(\lambda_{1}-\lambda_{2}\right) \eta_{1}^{i} \eta_{3}^{i}-}} \\
\frac{\frac{(\tau+1)}{2}\left(\eta_{1}^{i}\right)^{3}}{\lambda_{3} \eta_{3}^{i}+K_{\tau}\left(\eta_{1}^{i}\right)^{2}}
\end{array}\right]}
\end{aligned}
$$

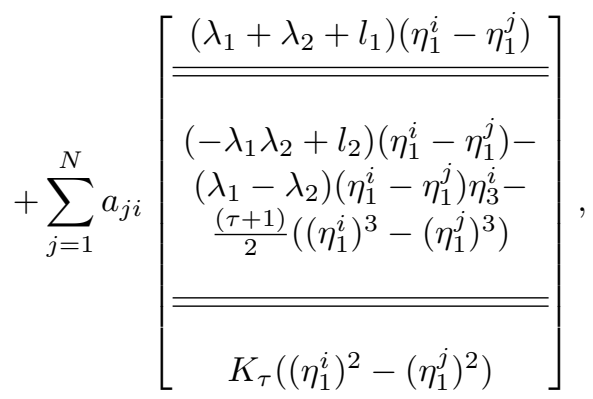

with a possibly non-symmetric binary adjacency matrix

$$
A=\left[\begin{array}{cccc}
a_{11} & a_{12} & \cdots & a_{1 N} \\
a_{21} & \ddots & \cdots & a_{2 N} \\
\vdots & \vdots & \ddots & \vdots \\
a_{N 1} & a_{N 2} & \cdots & a_{N N}
\end{array}\right] .
$$

Being the particular case with $N=2$, Theorem 3.4 suggests a general hypothesis that the synchronization between coupling nodes of CN (12), at least semi-globally, does not depend on the topology of its connections, as long as the corresponding $\mathrm{CN}$ remains synchronizable. In particular, for $\mathrm{CN}$ with $N$ nodes the claim 1 of Theorem 3.4 gives straighforwardly the following result.

Theorem 3.5. [Čelikovský et al., 2013] Consider CN (12) that is synchronizable and minimal. Then it can be globally exponentially synchronized.

\section{Auxiliary System Approach}

In this section, we repeat some knowledge about auxiliary system approach to detect the synchronization in the DCNs. Auxiliary system approach to detect the generalized synchronization between unidirectional coupled chaotic systems was introduced by [Abarbanel et al., 1996]. Fig. 2 illustrates the scheme of this method. This method uses the identical copy of the response system that is influenced by the same driving signal. The auxiliary system approach is useful for the detection of the generalized synchronization in the unidirectional coupled spatiotemporal systems [Rogers et al., 2004], laser systems [Jungling et al., 2019], microwave electronic systems [Plotnikova and Moskalenko, 2019], etc. In [Kocarev and Parlitz, 1996] the necessary and sufficient conditions for the occurrence of GS between unidirectional interconnected dynamical systems were analyzed. These conditions are based on the existence of the asymptotically stable limit set of the response system. Further, the application of auxiliary system approach for the GS regime detection was extended to bidirectional coupled dynamical systems in [Zheng et al., 2002]. This method has been widely implemented during the last decade to detect the GS regime in complex networks. Moreover, [Moskalenko et al., 2013] highlights that auxiliary system approach cannot be used correctly to detect the generalized synchronization regime in both the oscillators and networks of nonlinear elements with the mutual type of coupling. Therefore, the auxiliary system approach is insufficient for the detection the generalized regime in oriented complex networks. Recently, the essential conditions for the application of auxiliary system approach for detection of the GS regime in CN with directional coupling were reported in [Zhou et al., 2017]. Namely, it was concluded in [Zhou et al., 2017] that the auxiliary system approach is effective only when there is no path from nodes to their driving neighbors. The ring topology of the complex network does not respect the above condition, because there always exists a path from each node to their driving neighbor. In other words, the aforementioned condition satisfies only the topology of $\mathrm{CN}$ described by the directed acyclic graph (DAG), the so-called DAG network [Zhou et al., 2017], i.e. adjacency matrix $A$ of complex network is an upper triangular matrix. Using the terminology of Definition 2.1 , the auxiliary system approach is effective if $\mathrm{CN}$ has a master node with in-degree 0 which directly or indirectly influences all other nodes in the complex network. Therefore, an open question is how to apply the auxiliary system approach to detect the GS in DCCN. As we noticed before in [Čelikovský et al., 2013], DCCN is always synchronizable. Therefore, it is a challenging task to study possibility to apply auxiliary system approach to detect the GS in DCCN. Such a task is addressed in the next section using numerical experiments.

\section{Numerical Simulations}

In [Zhou et al., 2017] authors demonstrated that the auxiliary system approach is effective to be applied to detect the GS in CN with master node and without loops. In this section it is shown experimentally that the auxiliary system approach can be used for the detection the GS in $\mathrm{CN}$ with ring topology. Fig. 3 illustrates the application of auxiliary system approach to the 3-node complex network with ring topology and unidirectional coupling. Three extra nodes with the same structure like permanent nodes and the same control parameters need 


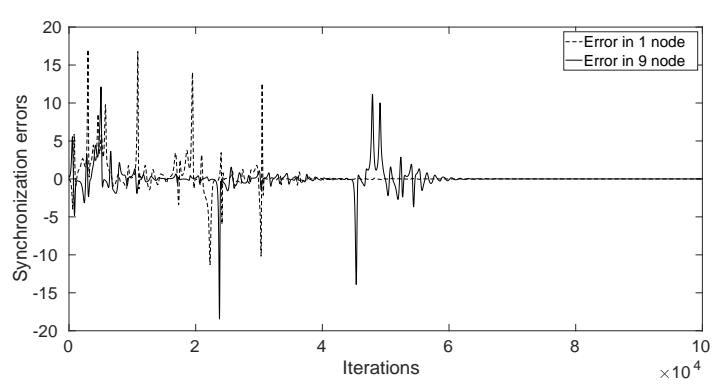

Figure 5. Synchronization errors between permanent and auxiliary nodes in 10-node cyclic complex network. Errors in the first and the ninth nodes are illustrated.

to be added to the original complex network for the detection the GS via this method. Further, the application of the auxiliary system approach for the detection of the GS in cyclic complex network illustrated in Fig. 1 will be analyzed. Here, directed cyclic complex network consists of 10 nodes being the generalized Lorenz system (12) with the same parameters $\lambda_{1}=8, \lambda_{2}=-16$, $\lambda_{3}=-1 ; a_{i j}$ are defined by adjacency matrix $A(13)$, $l_{1}, l_{2}=-5$ and by different control parameters $\tau_{i}(i$ is a number of node): $\tau_{1}=0.02, \tau_{2}=0.04, \tau_{3}=0.06$, $\tau_{4}=0.08, \tau_{5}=0.1, \tau_{6}=0.12, \tau_{7}=0.14, \tau_{8}=0.16$, $\tau_{9}=0.18, \tau_{10}=0.2$. Initial condition is taken as: $0 \leq\left[\eta_{1}^{i}, \eta_{2}^{i}, \eta_{3}^{i}\right]^{\top} \leq 1$ and $A$ is:

$$
A=\left[\begin{array}{llllllllll}
0 & 1 & 0 & 0 & 0 & 0 & 0 & 0 & 0 & 0 \\
0 & 0 & 1 & 0 & 0 & 0 & 0 & 0 & 0 & 0 \\
0 & 0 & 0 & 1 & 0 & 0 & 0 & 0 & 0 & 0 \\
0 & 0 & 0 & 0 & 1 & 0 & 0 & 0 & 0 & 0 \\
0 & 0 & 0 & 0 & 0 & 1 & 0 & 0 & 0 & 0 \\
0 & 0 & 0 & 0 & 0 & 0 & 1 & 0 & 0 & 0 \\
0 & 0 & 0 & 0 & 0 & 0 & 0 & 1 & 0 & 0 \\
0 & 0 & 0 & 0 & 0 & 0 & 0 & 0 & 1 & 0 \\
0 & 0 & 0 & 0 & 0 & 0 & 0 & 0 & 0 & 1 \\
1 & 0 & 0 & 0 & 0 & 0 & 0 & 0 & 0 & 0
\end{array}\right] .
$$

Ten auxiliary nodes with the same parameters $\tau_{i}$ like permanent nodes and different initial condition $0 \leq$ $\left[\eta_{1}^{i}, \eta_{2}^{i}, \eta_{3}^{i}\right]^{\top} \leq 1$ were added and connected to the permanent nodes in the master-slave configuration in the same way like Fig. 3 illustrates.

The synchronization errors between ten permanent coupled nodes of the analyzed complex network are illustrated in Fig. 4 (top subfigure). The level of the synchronization errors between permanent nodes of the complex network after some transient time is still big enough. Nevertheless, the synchronization errors between permanent and auxiliary nodes tend rapidly to zero, see Fig. 4 (bottom subfigure). Fig. 5 illustrates the synchronization errors between permanent and auxiliary systems of the first and the ninth nodes. These two nodes have a more unstable behavior in the analyzed cyclic complex network. But, it is shown that it is possible to use the auxiliary system approach to detect the GS in DCCN.

\section{Conclusions and Outlooks}

In this paper, auxiliary system approach was used to detect the generalized synchronization in the directed cyclic complex networks. The motivation of this research is to check the applicability of the auxiliary system approach for the detection of the GS in a complex network with ring topology. The auxiliary system approach allows detecting the GS in this kind of complex network. Using the auxiliary system approach to detect the GS in a complex network with bidirectional connections is still an open question. In our future research, we will focus on this problem.

\section{Acknowledgements}

This work has been supported by the Czech Science Foundation through the research grant No. 19-05872S.

\section{References}

Abarbanel, H. D. I., Rulkov, N. F., and Sushchik, M. M. (1996). Generalized synchronization of chaos: The auxiliary system approach. Physical Review E, 53(5), pp. 4528-4535.

Afraimovich, V. S., Verichev, N. N., and Rabinovich, M. I. (1986). Stochastic synchronization of oscillation in dissipative systems. Radiophysics and Quantum Electronics, 29(9), pp. 795-803.

Andrievsky, B. (2016). Numerical evaluation of controlled synchronization for chaotic Chua systems over the limited-band data erasure channel. Cybernetics and Physics, 5 (02), pp. 43-51.

Andrievsky, B., Fradkov, A. L., and Liberzon, D. (2017). Robust observers and Pecora-Carroll synchronization with limited information. In 2017 IEEE 56th Annual Conference on Decision and Control (CDC), Dec, pp. 2416-2421.

Boccaletti, S., Kurths, J., Osipov, G., Valladares, D., and Zhou, C. (2002). The synchronization of chaotic systems. Physics reports - review section of physics letters, 366 (1-2), pp. 1-101.

Boccaletti, S., Pisarchik, A., del Genio, C., and A.Amann (2018). Synchronization: From Coupled Systems to Complex Networks. Cambridge University Press.

Čelikovský, S. and Chen, G. (2002). On a generalized Lorenz canonical form of chaotic systems. International Journal of Bifurcation and Chaos, 12 (08), pp. 1789-1812.

Čelikovský, S. and Chen, G. (2005). Secure synchronization of a class of chaotic systems from a nonlinear observer approach. IEEE Transactions on Automatic Control, 50 (1), pp. 76-82.

Čelikovský, S. and Lynnyk, V. (2016). Message embedded chaotic masking synchronization scheme based on 
the generalized Lorenz system and its security analysis. International Journal of Bifurcation and Chaos, 26 (08), pp. 1650140.

Čelikovský, S., Lynnyk, V., and Chen, G. (2007). Robust structural synchronization in dynamical complex networks. In Proceedings of 7th IFAC Symposium on Nonlinear Control Systems, pp. 289-294.

Čelikovský, S., Lynnyk, V., and Chen, G. (2013). Robust synchronization of a class of chaotic networks. Journal of the Franklin Institute, 350 (10), pp. 2936-2948.

Chen, G. and Dong, X. (1998). From Chaos to Order. WORLD SCIENTIFIC.

Chen, G., Wang, X., and Li, X. (2014). Fundamentals of Complex Networks: Models, Structures and Dynamics. Wiley.

Erdös, P. and Rényi, A. (1960). On the evolution of random graphs. Publications of the Mathematical Institute of the Hungarian Academy of Sciences, 5, pp. 17-61.

Fujisaka, H. and Yamada, T. (1983). Stability theory of synchronized motion in coupled-oscillator systems. Progress of Theoretical Physics, 69 (1), pp. 32-47.

Jungling, T., Porte, X., Oliver, N., Soriano, M. C., and Fischer, I. (2019). A unifying analysis of chaos synchronization and consistency in delay-coupled semiconductor lasers. IEEE Journal of Selected Topics in Quantum Electronics, 25 (6), pp. 1-9.

Khramenkov, V., Dmitrichev, A., and Nekorkin, V. (2019). Dynamics and stability of two power grids with hub cluster topologies. Cybernetics and Physics, 8 (1), pp. 29-33.

Klinshov, V. and Nekorkin, V. (2017). Event-based simulation of networks with pulse delayed coupling. Chaos: An Interdisciplinary Journal of Nonlinear Science, 27 (10), pp. 101105.

Kocarev, L. and Parlitz, U. (1996). Generalized synchronization, predictability, and equivalence of unidirectionally coupled dynamical systems. Physical Review Letters, 76 (11), pp. 1816-1819.

Mainieri, R. and Rehacek, J. (1999). Projective synchronization in three-dimensional chaotic systems. Physical Review Letters, 82 (15), pp. 3042-3045.

Moskalenko, O., Koronovskii, A., Hramov, A., Zhuravlev, M., and Jaimes-Reategui, R. (2017). Residence time distributions for coexisting regimes of bistable dynamical systems subjected to noise influence. $C y$ bernetics and Physics, 6, pp. 97-102.

Moskalenko, O. I., Koronovskii, A. A., and Hramov, A. E. (2013). Inapplicability of an auxiliary-system approach to chaotic oscillators with mutual-type coupling and complex networks. Physical Review E, 87 (6), pp. 064901.

Parlitz, U. (2012). Detecting generalized synchronization. Nonlinear Theory and Its Applications, IEICE, 3 (2), pp. 113-127.

Pecora, L. M. and Carroll, T. L. (1990). Synchronization in chaotic systems. Phys. Rev. Lett., 64 (8), pp. 821-
824.

Pikovsky, A. S. (1984). On the interaction of strange attractors. Zeitschrift für Physik B Condensed Matter, 55 (2), pp. 149-154.

Plotnikov, S. A. and Fradkov, A. L. (2019). On synchronization in heterogeneous FitzHugh-Nagumo networks. Chaos, Solitons \& Fractals, 121, pp. 85-91.

Plotnikova, A. D. and Moskalenko, O. I. (2019). Specificities of generalized synchronization in delayed systems. Technical Physics Letters, 45 (6), pp. 560-562.

Rehák, B. and Lynnyk, V. (2019). Decentralized networked stabilization of a nonlinear large system under quantization. In Proceedings of the 8th IFAC Workshop on Distributed Estimation and Control in Networked Systems (NecSys 2019), September, pp. 1-6.

Rehák, B. and Lynnyk, V. (2019). Network-based control of nonlinear large-scale systems composed of identical subsystems. Journal of the Franklin Institute, 356 (2), pp. 1088-1112.

Rehák, B. and Lynnyk, V. (2019). Synchronization of symmetric complex networks with heterogeneous time delays. In 22nd International Conference on Process Control (PC19), IEEE, June, pp. 68-73.

Rogers, E. A., Kalra, R., Schroll, R. D., Uchida, A., Lathrop, D. P., and Roy, R. (2004). Generalized synchronization of spatiotemporal chaos in a liquid crystal spatial light modulator. Physical Review Letters, $93(8)$.

Rosenblum, M. G., Pikovsky, A. S., and Kurths, J. (1996). Phase synchronization of chaotic oscillators. Physical Review Letters, 76 (11), pp. 1804-1807.

Rosenblum, M. G., Pikovsky, A. S., and Kurths, J. (1997). From phase to lag synchronization in coupled chaotic oscillators. Physical Review Letters, 78 (22), pp. 4193-4196.

Rulkov, N. F., Sushchik, M. M., Tsimring, L. S., and Abarbanel, H. D. I. (1995). Generalized synchronization of chaos in directionally coupled chaotic systems. Phys. Rev. E, 51 (2), pp. 980-994.

Shilnikov, A. L., Shilnikov, L. P., and Turaev, D. V. (1993). Normal forms and Lorenz attractors. International Journal of Bifurcation and Chaos, 03(05), pp. 1123-1139.

Shilnikov, L. P. (1968). On the generation of a periodic motion from trajectories doubly asymptotic to an equilibrium state of saddle type. Mathematics of the USSRSbornik, 6 (3), pp. 427-438.

Zheng, Z., Wang, X., and Cross, M. C. (2002). Transitions from partial to complete generalized synchronizations in bidirectionally coupled chaotic oscillators. Physical Review E, 65 (5).

Zhou, J., Chen, J., Lu, J., and Lu, J. (2017). On applicability of auxiliary system approach to detect generalized synchronization in complex network. IEEE Transactions on Automatic Control, 62 (7), pp. 34683473. 